\title{
Etude des surfaces foliaires des arbres dépérissants
}

\author{
J.P. Garrec ${ }^{1}$, C. Kerfourn ${ }^{1}$ et E. Laitat ${ }^{2}$
}

1 INRA, Centre de Recherches Forestières, Laboratoire d'Etude de la Pollution Atmosphérique, Champenoux, 54280 Seichamps, France, et

2 Faculté des Sciences Agronomiques de l'Etat, Département de Biologie Végétale, 5800 Gembloux, Belgique

\section{Introduction}

Le phénomène du dépérissement des forêts se caractérise par deux perturbations histologiques foliaires: des carences minérales et des altérations des cires épicuticulaires (Huttunen et Laine, 1983).

Ces deux perturbations résulteraient, selon l'hypothèse qui implique fortement la pollution atmosphérique dans le dépérissement forestier, de modifications de la surface foliaire (cuticule et cires) principalement sous l'effet de l'ozone et du dépôt acide (sec et humide). Cette carence qui concerne surtout les éléments $\mathrm{K}, \mathrm{Ca}$ et $\mathrm{Mg}$, proviendrait alors d'un plus grand lessivage des ions de la feuille par la pluie ou le brouillard parallèlement aux altérations de la barrière cuticulaire.

\section{Altérations de la cuticule}

Trois types de mécanismes pourraient intervenir simultanément au niveau de la cuticule pour expliquer ces carences: 1) une augmentation des échanges cationiques au travers des surfaces cuticulaires suite à l'acidité des pluies; 2) une augmentation de la perméabilité des membranes cellulaires sous-jacentes sous l'effet de l'ozone essentiellement et pour une moindre part sous l'effet de l'acidité; 3) une augmentation de la perméabilité suite à des modifications physicochimiques induites par l'ozone et les pluies acides.

Dans le premier mécanisme, les effets du $\mathrm{pH}$ sur la perméabilité des cuticules aux ions sont bien connus (McFarlane et Berry, 1974). II a été démontré également que les feuilles sont capables, dans une certaine mesure, de neutraliser sur leur surface (par exsudation d'ions $\mathrm{K}, \mathrm{Ca}$ et $\mathrm{Mg}$ ) l'acidité de la pluie (Adams et Hutchinson, 1984).

Dans le deuxième mécanisme, les effets de l'ozone sur les cellules et en particulier sur les perméabilités membranaires ont fait l'objet de plusieurs travaux (Chimilis et Heath, 1975).

Le troisième mécanisme est souvent signalé dans les études sur le dépérissement des forêts, pour expliquer les 
carences (Elstner et Osswald, 1984). Un travail récent a montré qu'effectivement, sur les cuticules en place, il existe un effet des pluies acides sur la quantité et la nature des cires, mais aussi sur l'épaisseur et l'ultrastructure de la cuticule (Percy et Baker, 1987). Cependant, Ziegler citant les travaux de Lendzian signale que les cuticules sont pratiquement imperméables à l'ozone et que ce polluant ne semble pas affecter leur perméabilité à l'eau (Ziegler, 1987).

Pour vérifier la validité de ce mécanisme, nous avons soumis des cuticules isolées de feuilles de houx (llex aquifolium) à de l'ozone et à une solution acide, séparé- ment et conjointement. Les résultats ont montré que si l'on prenait des cuticules isolées de feuilles matures, les perméabilités à l'eau et aux ions ne sont pratiquement pas modifiées sous l'effet d'une solution acide ou de l'ozone (Garrec et Kerfourn, 1988) (cf. Fig. 1).

A partir de ces résultats, nous ne pensons pas que la pollution atmosphérique (ozone + dépôt acide) ait un effet direct notable sur la cuticule. Par contre, par son effet sur le métabolisme général de la plante, la pollution pourrait modifier indirectement les propriétés physicochimiques de la cuticule lorsqu'elle se met en place sur les feuilles en formation.

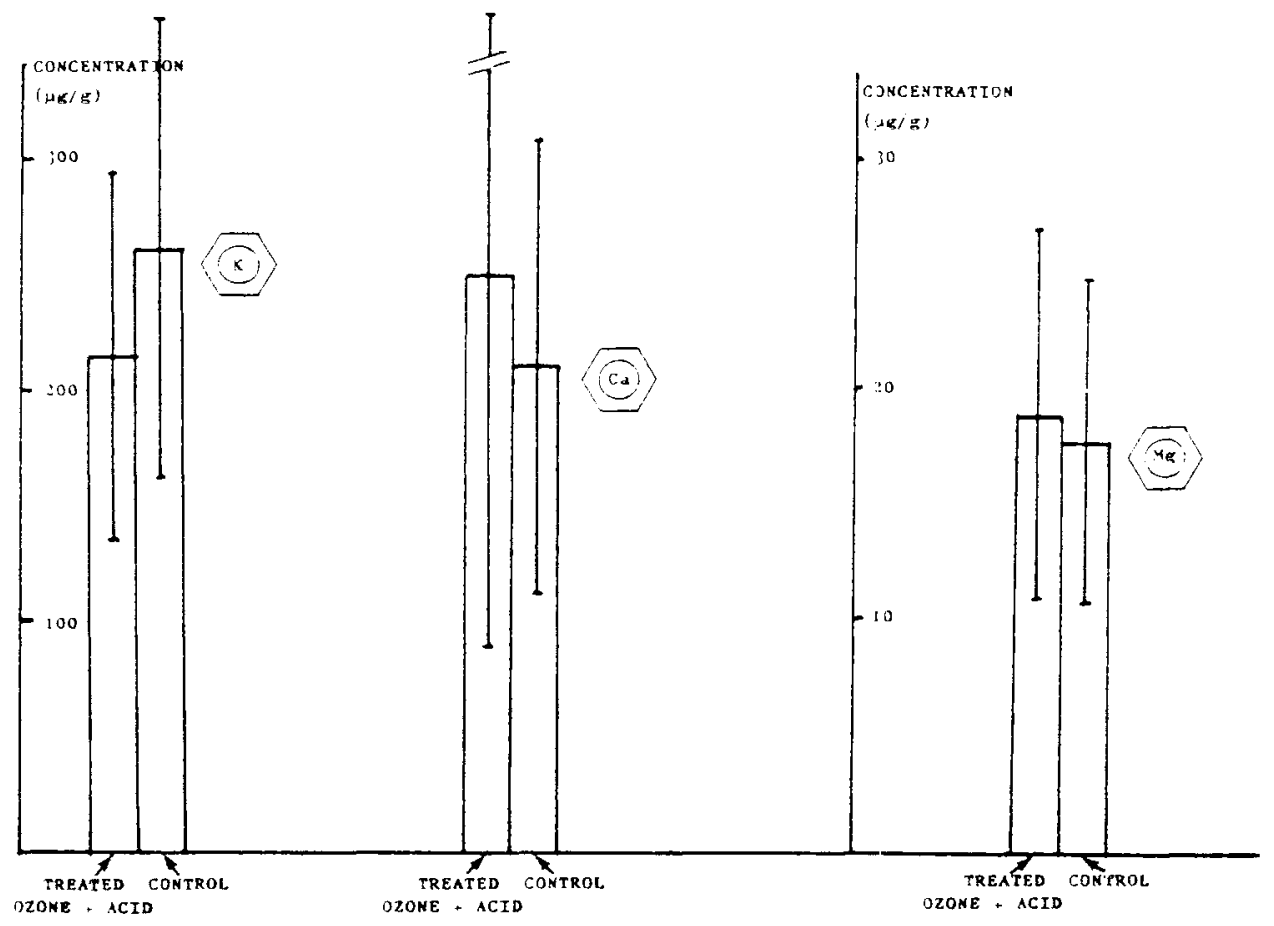

Fig. 1. Perméabilité aux ions de cuticules isolées (houx). Les concentrations en ions dans le liquide donneur sont respectivement : $\mathrm{Ca}=20 \mu \mathrm{g} / \mathrm{g}, \mathrm{Mg}=3 \mu \mathrm{g} / \mathrm{g}, \mathrm{K}=20 \mu \mathrm{g} / \mathrm{g}$. Les concentrations en ions dans le liquide receveur ont été mesurées au bout de $7 \mathrm{j}$. 
Cette "malformation" de la cuticule pourrait provenir: 1) d'un phénomène de vieillissement accéléré au niveau de la feuille déclenché par le "stress pollution"; 2) de la présence d'une carence minérale foliaire.

Pour vérifier la première hypothèse, nous avons étudié le lessivage des ions dans des aiguilles de sapins sains et de sapins dépérissants, par trempage de celles-ci dans de l'eau distillée (mesure de conductivité électrique de la solution). Les résultats ont montré que dans les aiguilles de sapins sains, le lessivage des ions augmente avec l'âge, et que dans les sapins dépérissants, celui-ci augmente avec le degré de dépérissement (Boiteux, 1988) (cf. Fig. 2). Au niveau du lessivage des ions, l'expérience semble donc suggérer un parallèle entre le dépérissement forestier et un phénomène de senescence précoce dans les aiguilles (la classe III correspondrait à un vieillissement accéléré de 2 ans environ).

\section{Altérations des cires épicuticulaires}

A partir d'observations en microscopie électronique à balayage, 2 sortes d'altérations sont généralement signalées: 1) une diminution (ou une érosion) de la quantité de cires épicuticulaires; 2) une modification de la forme des cires substomatiques (agglomération, fusion, formation d'un bouchon) (Laitat, 1987).

De nombreuses recherches ont été effectuées pour déterminer à partir de simulations en laboratoire (action de l'ozone, action des pluies acides) l'origine des modifications de ces cires (Grill et al., 1987; Gunthardt-Goerg et Keller, 1987). Nous pensons que comme pour les cuticules, ces altérations morphologiques ne proviennent pas d'effets directs des pol- luants, ni ne sont spécifiques de ceux-ci. Elles seraient comme précédemment dûes à une senescence accélérée au niveau du métabolisme des aiguilles dans les arbres dépérissants.

En effet, à partir de nombreuses observations en microscopie électronique à balayage, nous avons constaté que l'évolution de la forme des cires sur des aiguilles saines en cours de leur vieillissement est identique à l'évolution des cires sur des aiguilles d'arbres de plus en plus dépérissants.

Ces observations sont confirmées expérimentalement lorsque l'on mesure l'angle de contact des gouttes d'eau sur les surfaces foliaires. Les résultats ont montré que sur les aiguilles saines, l'angle de contact diminue avec le vieillissement (augmentation de la mouillabilité de la surface). Sur les aiguilles d'arbres dépérissants nous constatons de même une diminution de l'angle de contact avec le dépérissement (Boiteux, 1987) (cf. Fig. 3).

Parallèlement lorsque l'on a soumis de cuticules isolées à de l'ozone et à de l'acide, nous n'avons pas constaté de modifications significatives de l'angle de contact entre les cuticules témoins et les cuticules traitées. Ceci indiquerait bien que ces polluants n'entraînent pas de changements importants directs au niveau de la structure de la surface (forme, composition chimique) (Garrec et Kerfourn, 1988).

Plusieurs travaux ont été consacrés aux effets des polluants atmosphériques $\left(\mathrm{O}_{3}\right.$, $\mathrm{SO}_{2}$ ) sur la composition chimiques des cires épicuticulaires (Huttunen et al., 1985; Cape, 1986; Goerg-Gunthardt et Keller, 1987). Par contre peu d'études ont abordé ce problème dans les arbres dépérissants in situ (Simmleit et Schulten, 1988). Généralement, il a été constaté de faibles variations de la composition chimique des cires dans les arbres dépérissants et que ces 
550 s

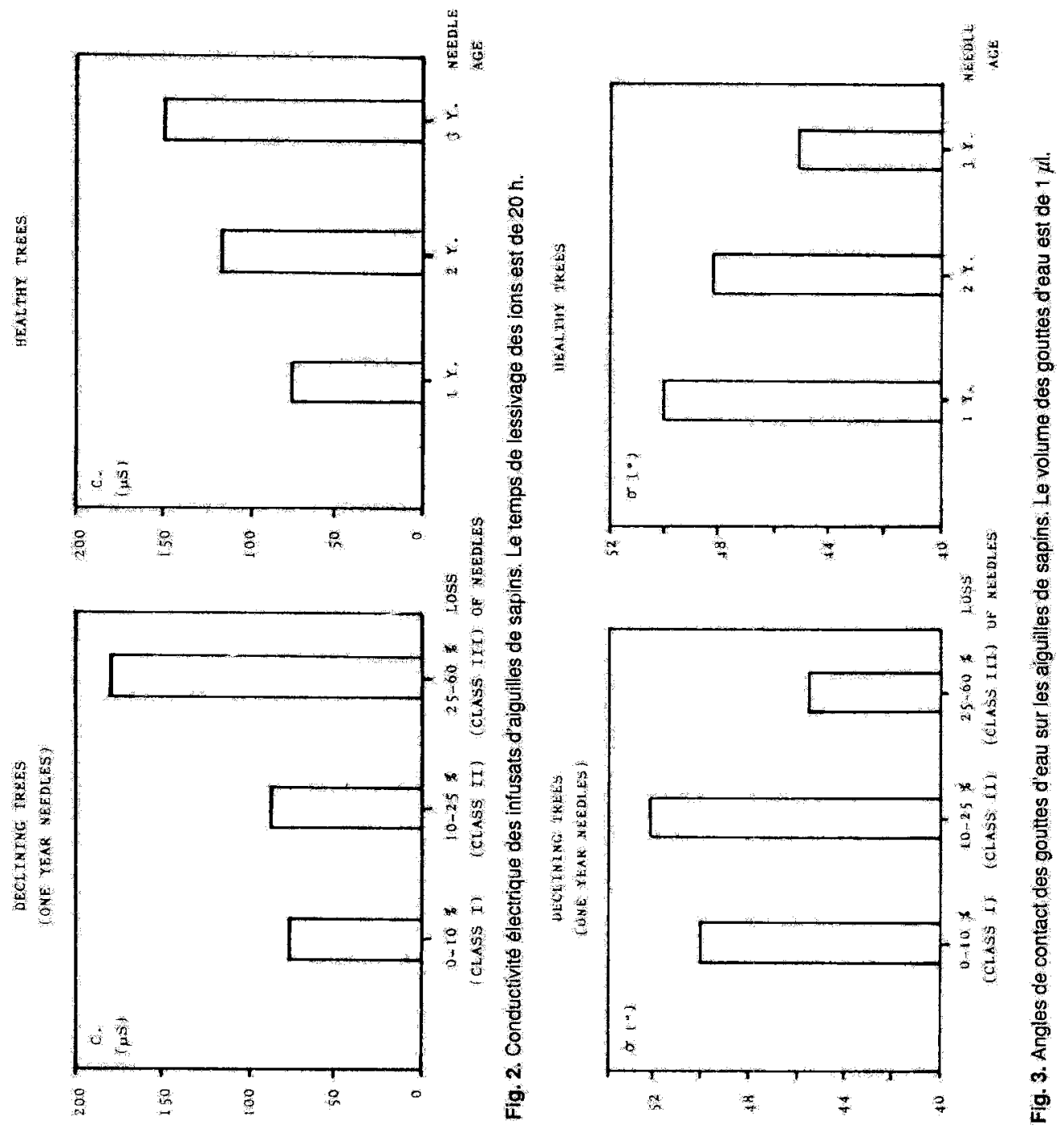


variations sont sensiblement identiques à celles que l'on observe dans les aiguilles saines au cours de leur vieillissement. tiellement pour origine des problèmes de nutrition minérale au niveau du sol ou des systèmes racinaires. Dans ce cas, la plante ne pourrait plus compenser ses pertes foliaires.

\section{Conclusions}

A partir de nos travaux sur les cuticules et sur les cires exposées à de l'ozone ou à des solutions acides et des données de la littérature, nous tirons les conclusions suivantes: 1) il ne semble pas que les polluants atmosphériques soupçonnés d'être à l'origine du dépérissement (ozone, dépôt acide) aient des effets spécifiques et directs sur les surfaces foliaires; 2) le dépérissement résulterait de la conjonction à un moment donné d'un certain nombre de stress parmi lesquels semblent se dégager le stress climatique (sécheresse) mais aussi le stress pollution atmosphérique (vraisemblablement composé d'un ensemble de composants agissants à des niveaux différents: dépôt acide sec et humide, ozone, micropolluants organiques, plomb, etc.); 3) tous ces stress agiraient à un niveau relativement faible, ce qui se traduirait sur le métabolisme des arbres avant tout par la mise en route d'un processus de senescence précoce (Garrec et al., 1988); 4) nous ne savons pas si la carence minérale foliaire qui s'observe conjointement au processus de senescence et une des causes, une des conséquences ou est indépendante de ce phénomène. Mais l'augmentation de la perméabilité des surfaces foliaires aux ions sous l'effet du vieillissement est trop faible, à notre avis, pour expliquer d'une part cette carence et d'autre part les fortes concentrations d'ions dans les pluviolessivats ("leaching"). Nous pensons qu'une partie importantes des ions contenus dans les pluviolessivats provient du dépôt sec sur la surface foliaire. Quant aux carences minérales foliaires, elles auraient essen-

\section{Références}

Adams C.M. \& Hutchinson T.C. (1984) A comparison of the ability of leat surfaces of three species to neutralize acidic rain drops. New Phytol. 97, 463-478

Boiteux E. (1988) Surfaces foliaires d'arbres dépérissants: étude de la conductivité électrique des solutions de lessivage, et de l'angle de contact des gouttes d'eau. Rapport interne.

Cape J.N. (1986) Effects of air pollution on the chemistry of surface waxes of Scots pine. Water Air Soil Pollut. 31, 393-399

Chimiklis P.E. \& Heath R.L. (1975) Ozone-induced loss of intracellular potassium ion from Cholorella sorokiniana. Plant Physiol. 56, 723727

Elstner E.F. \& Osswald W. (1984) Fichtensterben in 'reinluftgebieten': structurresistenzverlust. Naturwiss. Rundsch. 37, 52-61

Garrec J.P. \& Kerfourn C. (1989) Effets des pluies acides et de l'ozone sur la perméabilité à l'eau et aux ions de cuticules isolées. Implication dans le phénomène de dépérissement. Environ. Exp. Bot. 29, 215-228

Garrec J.P., Richardin I., Le Maout L., Rose C., Gerard B. \& Mathieu Y. (1989) Etude du pH et du pouvoir tampon des aiguilles de sapins (Abies alba L.) et d'épicéas (Picea abies L.) dépérissants. Rev. For. Fr. 46, 55-69

Goerg-Gunthardt M.S. \& Keller T. (1987) Picea abies: needle surface after fumigation with low concentrations of $\mathrm{SO}_{2}$ or $\mathrm{O}_{3}$. C.E.C. Air Pollut. Res. Rep. 4, 159-160

Grill D., Pfeifhofer H., Halbwachs G. \& Waltinger $H$. (1987) Investigations on epicuticular waxes of differently damaged spruce needles. Eur. J. For. Pathol. 17, 246-255

Gunthardt-Goerg M.S. \& Keller T. (1987) Some effects of long-term ozone fumigation on Norway spruce. II. Epicuticular wax and stomata. Trees 1, 145-150 
Huttunen S. \& Laine K. (1983) Effects of airborne pollutants on the surface wax structure of Pinus sylvestris needles. Ann. Bot. Fenn. 20, 79-86

Huttunen S., Makela M. \& Karhu M. (1985) Effects of acid deposition on needle surfaces. In: Air Pollution and Plants, (Troyanowsky C., ed.), VCH, pp. 218-221

Laitat E. (1987) Criteria for early diagnosis of forest decline and simulation of its effect through a defoliation technique. C.E.C. Air Pollut. Res. Rep. 4, 210-218

McFarlane J.C. \& Berry W.L. (1974) Cation penetration through isolated leaf cuticules. Plant Physiol. 53, 723-727
Percy K.E. \& Baker E.A. (1987) Effects of simulated acid rain on production, morphology and composition of epicuticular wax and on cuticular membrane development. New Phytol. 107, 577589

Simmleit N. \& Schulten H.R. (1988) On the impact of acidic precipitation on the epicuticular wax of spruce needles. In: C.E.C. Air Pollution and Ecosystems. (Mathy P., ed.), D. Reidel, pp. 546-553

Ziegler H. (1987) Interactive effects of dry and wet deposition on structure and function of canopies, in particular interactive effects of $\mathrm{SO}_{2}, \mathrm{NO}_{x}$ and $\mathrm{O}_{3}$. C.E.C. Air Pollut. Res. Rep. 4. $102-109$ 